\title{
CLASSIFICAÇÃO E ELIMINAÇÃO DOS RUÍDOS EM IMAGENS HIPERESPECTRAIS PELA ANÁLISE SEQÜENCIAL DA TRANSFORMAÇÃO POR FRAÇÃO DE RUÍDO MÍNIMA
}

\author{
Osmar A. Carvalho Júnior, ${ }^{1}$ Ana P. F. Carvalho, ${ }^{2}$ Paulo R. Meneses ${ }^{3}$ e Renato F. Guimarães ${ }^{4}$ \\ Recebido em 3 dez., 2001 / Aceito em 20 ago., 2003 \\ Received Dec. 3, 2001 / Accepted Aug. 20, 2003
}

\begin{abstract}
Hyperspectral images are extremely susceptible to noise interferences which hinder mineral identification and quantification. Therefore, it is fundamental the use of techniques to minimize them. An efficient mathematical noise reducing procedure employs the Minimum Noise Fraction (MNF). The MNF procedure is derived from and similar to the Principal Component Analysis (APC). The main difference between them is that the MNF considers the noise while the PCA considers the data variation. It results in an ordering that reflects the image quality. The MNF transformation can be subdivided in four stages: a) obtaining a noise sample; b) formulation of a noise fraction index; $c$ ) implementation of a linear transformation function as P(A; and d) inversion of MNF considering only signs information. In the present work a methodology of MNF sequential steps is proposed using internal and external references of the image. As external reference it was used the dark reference collected during a flight simultaneously to the image acquisition. The internal reference was obtained by statistical techniques for the segmentation of the noise fraction in relation to the sign. Three types of noises can be defined by variance and correlation: a) uncorrelated noises with equal variance in all bands, b) highly correlated noises, and c) Noises with Unknown Covariance. Matrix. For the AVIRIS images, a sequential procedure of MNF can be accomplished with two stages: the first one eliminates the noises of intern reference and the second the relative external reference to the instrumental noise. That procedure allows to distinguish different types of noises and to eliminate them sequentially and efficiently.
\end{abstract}

Keywords: Remote sensing; image processing; hyperspectral; noise.

\section{RESUMO}

As imagens hiperespectrais são muito suscetíveis às interferências de ruídos, o que dificulta a identificação e a quantificação mineral. Portanto, torna-se fundamental 0 emprego de técnicas para minimizá-los. Um eficiente procedimento matemático para redução de ruídos é a Fração de Ruído Mínima (MNF). Esse método é derivado e análogo à transformação Análise de Componentes Principais, distinguindo-se por promover a maximização do ruído ao invés da variância dos dados, 0 que proporciona um ordenamento que reflete a qualidade das imagens. A transformação MNF pode ser subdividida em quatro etapas: a) obtenção de uma amostra do ruído; b) formulação de um índice de fracionamento do ruído; c) implementação de uma função de transformação linear no molde da APC, e d) inversão do MNF considerando apenas as informações de sinais. No presente trabalho é proposta uma metodologia de MNF seqüencial, utilizando referências internas e externas da imagem. Como referência externa foi utilizada a referência escura coletada durante 0 vôo simultaneamente à obtenção da imagem. A referência interna foi obtida por técnicas estatísticas para a segmentação da fração do ruído em relação ao sinal. Três tipos de ruídos podem ser definidos conforme a variância e correlação: a) ruídos não-correlacionados e com igual variância; b) ruídos altamente correlacionados; e c) ruídos com matriz de covariância desconhecida. Para as imagens AVIRIS pode-se esquematizar um procedimento seqüencial de MNF com duas etapas, sendo que, na primeira, são eliminados os ruídos de referência interna e na segunda, os de referência externa relativos ao ruído instrumental. Esse procedimento permite distinguir diferentes tipos de rúdos e eliminá-los seqüencialmente, de forma simples e eficiente.

Palavras-chave. Sensoriamento remoto; hiperespectral; processamento de imagem; ruído.

\footnotetext{
Departamento de Geografia - Universidade de Brasilia (UnB) - Campus Universitário Darcy Ribeiro, Asa Norte, 70910-900, Brasília, Distrito Federal. Tel.: (61) 3072474. E-mail: osmarir@unb.br 2 Departamento de Ecologia - Universidade de Brasília (UnB) - Campus Universitário Darcy Ribeiro, Asa Norte, 70910-900, Braślia, Distrito Federal. Fone: (61) 367-5001. Fax: (61) 2721909. Email: anapaula@unb.br

3 Departamento de Geologia Geral e Aplicada - Universidade de Braślia (UnB) - Campus Universitário Darcy Ribeiro, Asa Norte, 70910-900, Braślia, Distrito Federal. Fone (61) $348-2872$ Fax (61) 347-4062. E-mail: pmeneses@unb.br

4 Departamento de Geografia - Universidade de Brasília (UnB) - Campus Universitário Darcy Ribeiro, Asa Norte, 70910-900, Braślia, Distrito Federal. Fone: 3071859. Fax: (61) 2721909. E-mail: renatofg@unb.br
} 


\section{INTRODUÇÃOO}

A espectroscopia de imageamento introduziu uma nova fase de aquisiç̧ão de dados de sensoriamento remoto óptico. Essa tecnologia proporciona a obtenção de imagens em um grande número de bandas estreitas, amostrando o espectro eletromagnético de maneira praticamente contínua na faixa de 400-2500nm. Seu conceito operacional é apresentado na Figura 1. A alta resoluçãa espectral torna a informação de cada elemento de imagem (pixe) da cena próxima à obtida por meio de medições realizadas em laboratório e/ou campo (ABRAMS, 1986; VANE; GOETZ, 1988; KRUSE, 1988).

0 sensor AVIRIS - (Airborne Visible/InfraRed Imaging Spectrometer) -, desenvol-vido em 1983 por pesquisadores do Jet Propulsion laboratory (JPL/Califórnia) (VANE et al., 1988), foi o primeiro sistema hiperespectral a tornar-se operacional em 1989. Esse sensor, que normalmente adquire dados a partir de uma aeronave, foi trazido para 0 Brasil em 1995 na missão SCAR-B - (Smoke, Clouds and Radiation - Brasil), que teve como propósito avaliar efeitos atmosféricos. Essa atividade foi uma operação conjunta da NASA - (National
Aeronautics and Space Administration)-, INPE - (Instituto Nacional de Pesquisas Espaciais) - e AEB (Agência Espacial Brasileira) - (KAUFMAN et al., 1998).

Mesmo com a riqueza de informações dos dados provenientes de sensores hiperespectrais, deve-se considerar que, para obter informações precisas, tanto na identificação quanto, principalmente, na quantificação mineral, é fundamental eliminar ou minimizar a interferência de rú́dos. Portanto, a análise do ruído é fundamental para a aquisição de bons dados, pois a capacidade de detecção de alvos está intimamente relacionada à raz̃o sinal/ruído.

Neste trabalho enfatiza-se a técnica Fração de Ruído Mínima (Minimum Noise Fraction - MNF) -, método originado da Análise de Principais Componentes (APC). Esse método foi proposto por Green e outros (1988), sendo originalmente denominado de Fração de Ruído Máxima. No entanto, Boardman \& Kruse (1994) propuseram uma nova denominação do método como Fração de Ruído Mínima, considerando a ordem inversa da saída das componentes. Este método concilia tanto os procedimentos de segregação da componente ruído como também de redução da dimensionalidade dos dados, os quais o tornam uma

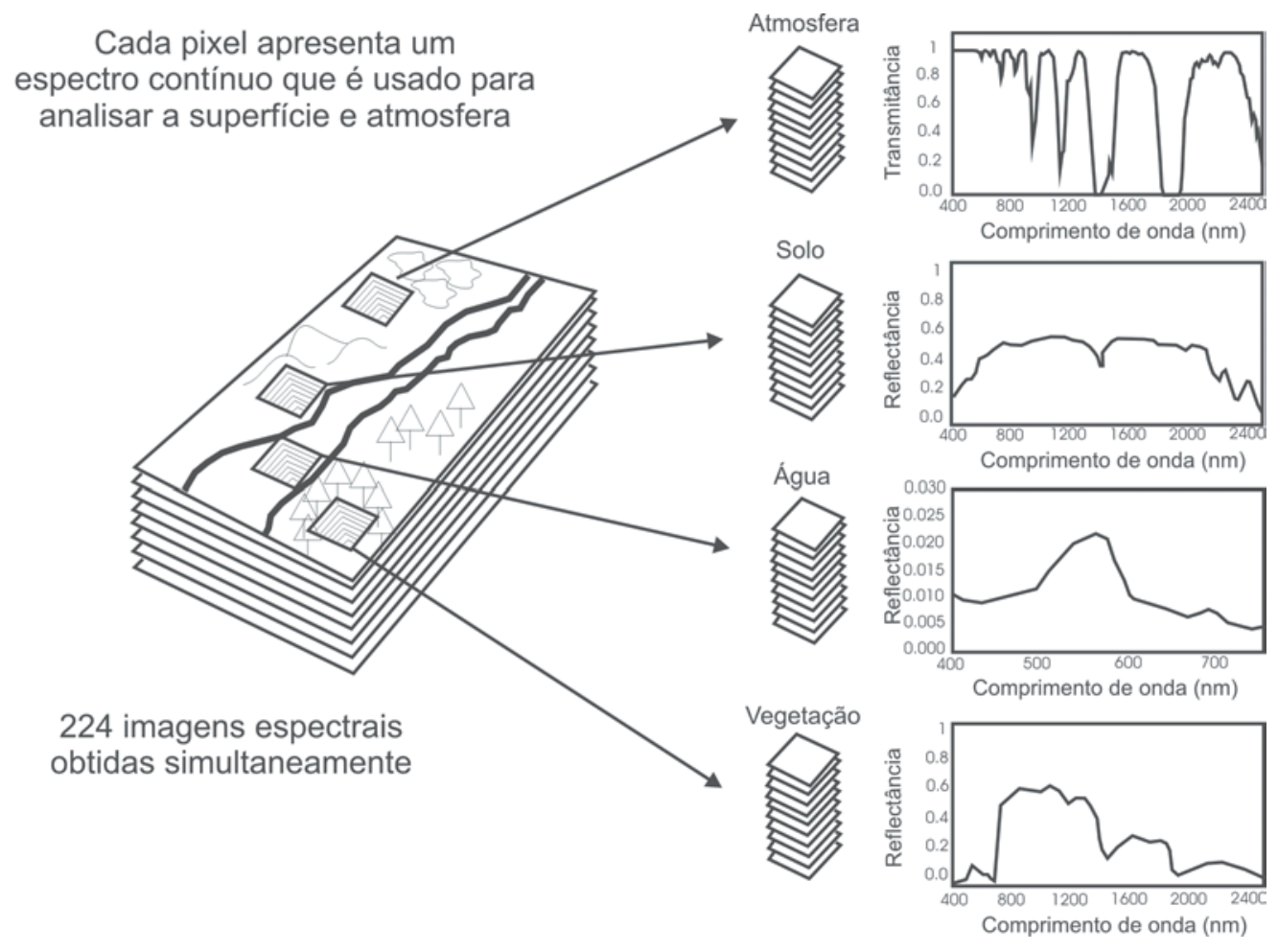

Figura 1 - Concepção de imagens hiperespectrais. Figure I - Conception of hyperspectral images. 

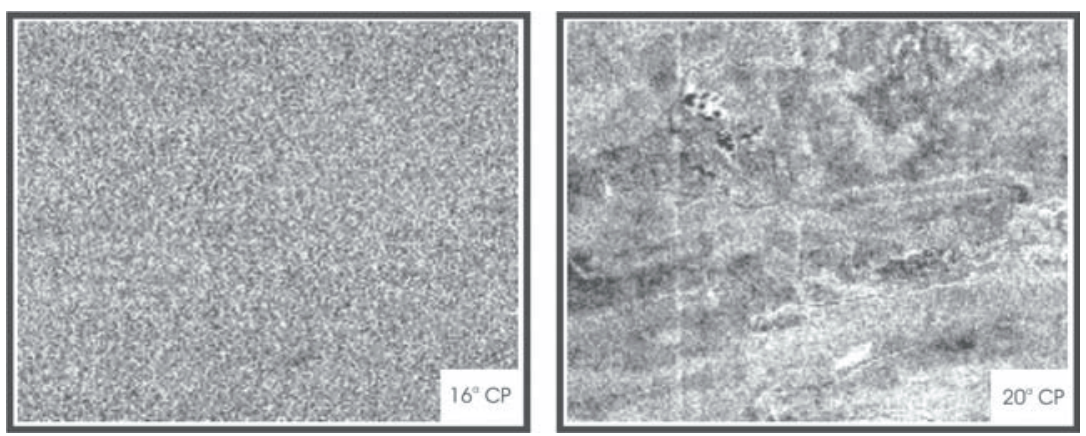

Figura 2 - Imagens relativas a $16^{a}$ e $20^{a}$ Principais Componentes do sensor AVIRIS da região de Niquelândia. Figure 2 - Images related to the $16^{\text {th }}$ to $20^{\text {th }}$ Principal Components of AVIRIS sensor in the region of Niquelandia.

importante fase no processamento digital para a identificação dos membros finais de uma imagem. No presente trabalho é proposta uma sequiência de etapas de MNF, visando o tratamento de ruídos em imagens hiperespectrais.

\section{Ordenação do ruído}

A transformação MNF é um procedimento estatístico no âmbito da APC. Com 0 advento da espectroscopia de imageamento, houve um aumento nos atributos que descrevem a imagem, tornando necessária a adequação da APC a um ambiente com maior número de variáveis e de ruído. Dessa forma, a transformação MNF é justamente um aprimoramento da APC para 0 ambiente hiperespectral.

Uma das grandes limitações da APC para a remoçãa do ruído é que nem sempre se observa nas componentes principais geradas um aumento da razão sinal/ruído de forma crescente em relação aos autovalores (GREEN et al., 1988; LEE et al., 1990). Essa tendência aumenta com a complexidade dos dados. Dessa forma, para as imagens multiespectrais pode-se, com freqüência, ter um ordenamento das componentes principais (CPS) conforme a intensidade do ruído. № entanto, para as imagens hiperespectrais, que consituem dados mais complexos, isso dificilmente ocorre. 0 tratamento por APC da imagem hiperespectral de Niquelândia (GO) demonstra claramente essa característica. Observa-se que a $16^{\circ} \mathrm{CP}$ apresenta um grau de ruído muito superior ao da 20॰ CP (Figura 2).

A vulnerabilidade da APC para a eliminação do ruído é resultante da priorização da variância no ordenamento dos dados, ao invés da razão sinal/ruído. Assim sendo, Green e outros (1988) propuseram 0 procedimento estatístico MNF, que enfoca o ruído em depreciação da variância do dado.

\section{Formulação do MNF}

0 procedimento matemático do MNF é derivado e análogo à transformação APC, distinguindo-se apenas quanto ao critério para a geração das componentes. 0 MNF promove a maximização do ruído ao invés da variância dos dados, 0 que proporciona um ordenamento que reflete a qualidade das imagens (GREEN et al., 1988).

0 desenvolvimento matemático pode ser dividido em quatro etapas: a) obtenção de uma amostra do ruído e cálculo de sua matriz de covariância; b) estabelecimento de um índice de fracionamento do ruído; c) implementação da função de transformação linear nos moldes da APC; e d) inversão do procedimento do MNF considerando apenas a fração do sinal.

\section{Obtenção de uma amostra do ruído}

A obtenção de uma amostra do ruído o MNF pode ser oriunda de uma referência interna ou externa à imagem

\section{Referência de ruído interna à imagem}

A amostra de ruído é proveniente da própria imagem, sendo utilizadas técnicas estatísticas para a segmentação da fração ruída do sinal. Considerando os dados multivariados de uma imagem (Z) com " $p$ " bandas $e$ " $x$ " amostras, temos que:

$\mathrm{Zi}(\mathrm{x}), \mathrm{i}=1, \ldots ., \mathrm{p}$

Considerando que o conjunto de dados é constituído por sinal e ruído, temos: 


\section{$\mathrm{Z}(\mathrm{x})=\mathrm{S}(\mathrm{x})+\mathrm{R}(\mathrm{x})$ \\ Estabelecimento de um Índice de Fracionamento do Ruído}

onde:

$\mathrm{S}(\mathrm{x})=$ componente do sinal, $\mathrm{e}$

$\mathrm{R}(\mathrm{x})=$ componente do ruído.

De forma análoga, a covariância dos dados também se apresenta segmentada em sinal e ruído:

$\operatorname{Cov}\{\mathrm{Z}(\mathrm{x})\}=\mathrm{S}=\mathrm{Ss}+\mathrm{Sr}$

onde:

Ss = matriz de covariância do sinal, e

$\mathrm{Sr}=$ matriz de covariância do ruído.

A filtragem e a APC são os principais métodos utilizados para se obter uma prévia segmentação dos dados em ruído e sinal e, conseqüentemente, de uma amostragem da componente ruído.

\section{Referência de Ruído Externa à Imagem}

A amostra de ruído é proveniente de medições de referência externa, como a referência escura ou de viagnetcoletada durante o vôo, simultaneamente à obtenção da imagem.

A referência escura (dark reference) demonstra o comportamento do ruído instrumental durante 0 evento de vôo. Alguns instrumentos de espectroscopia de imageamento registram referências escuras para cada linha da imagem. Lee e outros (1990) utilizaram dados de referência escura para a remoção de ruídos de imagens do Geophysical and Environmental Research (GER), com 64 canais, obtidas em novembro de 1987 durante uma missão na Austrália.

Considerando um conjunto de dados de referência escura (Zre) com " $x$ " amostras $e$ " $p$ " bandas, referentes a uma determinada imagem, pode-se estabelecer uma matriz de covariância do ruído:

$\operatorname{Cov}\{\operatorname{Zre}(\mathrm{x})\}=\operatorname{Ser}$

onde:

Ser $=$ matriz de covariância do ruído relativo à referência escura.
A partir da segmentação dos dados (sinal/ruído) pode-se estabelecer um índice que enfatize a fração ruído. Portanto, pode-se definir a seguinte relação para as " $i$ " bandas:

$\operatorname{IFR}=\operatorname{Var}\{\operatorname{Ri}(\mathrm{x})\} / \operatorname{Var}\{\operatorname{Zi}(\mathrm{x})\}$

Onde:

IFR = índice da fração ruído

$\operatorname{Var}\{\operatorname{Ri}(\mathrm{x})\}=$ variância do ruído

$\operatorname{Var}\{\operatorname{Zi}(\mathrm{x})\}=$ variância total dos dados

Dessa forma, estabelece-se uma matriz de covariância do índice de fracionamento do ruído nos mesmos moldes de uma matriz de covariância utilizada em uma APC (Figura 3).

\section{Implementação de uma Função de Transformação Linear nos Moldes da APC}

De caráter linear, a transformação MNF é expressa como:

$\mathrm{Yi}(\mathrm{x})=\operatorname{aTi} \mathrm{Z}(\mathrm{x}), \mathrm{i}=1 \ldots \mathrm{p}$

Eq. (6)

Usando argumento similar ao da APC, temos que ai representa os autovetores e o $\mathrm{Sr} / \mathrm{S}$ é 0 mi referente aos autovalores associados, que quivale à fração do ruído em Yi. Portanto, a partir dessa definição verificase, para a transformação MNF, um ordenamento dos autovalores de acordo com a qualidade da imagem, onde:

$\mathrm{m} 1{ }^{3} \mathrm{~m} 2{ }^{3} \mathrm{~m} 3{ }^{3} \quad \ldots .{ }^{3} \mathrm{mp}$

Eq. (7)

De acordo com a formulação matemática, a grande diferenç̧a do MNF para a APC é a substituição da matriz de covariância-variância dos dados pela matriz relativa cos índices de fracionamento de ruído. A utilização de uma ou outra matriz proporciona a maximização de um determinado tipo de informação.

A aplicação da transformação requer o conhecimento ou a estimativa da matriz de covariância do rúído, ou seja, os membros $\mathrm{Sr}$ e S. 0 membro Sé facilmente obtido a partir da matriz de covariância dos dados $Z(x)$, ou seja, da imagem em análise, enquanto o membro (Sr) 
pode ser estimado de diversas formas, dependendo do ruído previamente amostrado.

\section{Inversão do MNF considerando apenas as Informações de Sinais}

Após 0 emprego da transformação linear MNF efetua-se a separação da fração ruído do sinal. Como as imagens estão ordenadas de acordo com a qualidade, torna-se fácil realizar essa separação. Desta forma, considerando apenas as componentes relativas ao sinal, ou seja, desprezando as frações ruidosas, efetua-se o processo de inversão da transformação MNF. As imagens geradas com o processo de inversão apresentam espectros límpidos de ruído.

\section{TRANSFORMACC̃̃O MNF UTILIZANDO REFERÊNCIA DE RUÍDO INTERNA À IMAGEM}

0 procedimento para a prévia discriminação da fração ruído consiste no principal fator para a diversidade metodológica da técnica MNF. Há muitas formas para estimar o ruído e, conseqüentemente, sua matriz de covariância. Portanto, a primeira pergunta que deve ser feita é: que tipo de ruído se deseja minimizar? A partir da definição do tipo de ruído que se quer eliminar pode-se estabelecer uma metodologia de amostragem e correção.

Quando se utilizam referências externas à imagem, a componente do ruído já está discriminada e, portanto, pronta para ser empregada no MNF. Em contraposição, o emprego de referências de ruído internas requer procedimentos estatísticos, o que possibilita inúmeras abordagens que variam de acordo com ruído que se deseja enfatizar. Os procedimentos mais usuais para a segmentação do sinal-ruído em uma imagem são a filtragem e a APC, sendo esta última a mais empregada.

\section{MNF utilizando Filtragem Espacial}

Green e outros (1988) sugeriram uma técnica de filtragem denominada Fator de Autocorrelação Mínimo/Máximo (FAM) para 0 tratamento de ruídos do tipo sal e pimenta (salt-and-pepper noise), ou para outras formas de degradação de sinal, tais como a presença de linhas danificadas na imagem. 0 emprego de filtros espaciais baseia-se no fato de o sinal de um pixe/da imagem apresentar uma forte correlação com o sinal dos pixe/s vizinhos, enquanto o ruído apresenta uma fraca correlaçãa espacial. Dessa forma, os pixels ruidosos são localizados e isolados para o cálculo da matriz de covariância do ruído (Sr) e da matriz do índice da fração rú́do, proporcionando a maximização do ruído nos

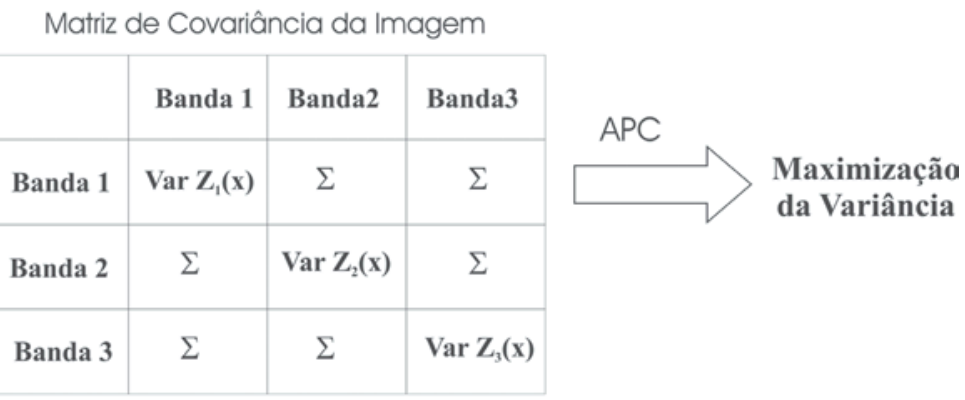

Matriz de Covariância do Índice de Ruído

\begin{tabular}{|c|c|c|c|c|}
\hline & Banda 1 & Banda2 & Banda3 & \multirow{7}{*}{ MNF } \\
\hline \multirow{2}{*}{ Banda 1} & $\operatorname{Var}\left\{R_{1}(x)\right\}$ & $\sum_{r}$ & $\Sigma_{r}$ & \\
\hline & $\operatorname{Var}\left\{Z_{1}(x)\right\}$ & $\bar{\Sigma}$ & $\bar{\Sigma}$ & \\
\hline \multirow{2}{*}{ Banda 2} & $\underline{\sum_{r}}$ & $\operatorname{Var}\left\{R_{2}(x)\right\}$ & $\sum_{r}$ & \\
\hline & $\Sigma$ & $\operatorname{Var}\left\{Z_{2}(x)\right\}$ & $\Sigma$ & \\
\hline \multirow[t]{2}{*}{ Banda 3} & $\underline{\Sigma_{r}}$ & $\sum_{r}$ & $\operatorname{Var}\left\{R_{3}(x)\right\}$ & \\
\hline & $\bar{\Sigma}$ & $\bar{\Sigma}$ & $\operatorname{Var}\left\{Z_{3}(x)\right\}$ & \\
\hline
\end{tabular}

Figura 3 - Comparação das matrizes de covariância utilizadas nas transformações APC e MNF. Figure 3 - Comparison of the covariance matrixes used in APC and MNF transformations. 
moldes da APC. No entanto, Lee e outros (1990) alertaram que essa metodologia só pode ser empregada para ruídos que apresentem diferenças com sua vizinhança detectáveis. Muitos dos ruídos de uma imagem hiperespectral são periódicos, correspondendo a vários pixels, 0 que, nesse caso, torna o FAM inadequado.

Outra tentativa para separar esse tipo de ruído é o emprego da transformação de Fourier. Rose (1989) utilizou-a em imagens AVIRIS obtendo bons resultados. Um grande inconveniente desse método é que cada banda tem de ser tratada individualmente, o que torna o processo muito moroso. No próximo tópico é proposto um procedimento simples e rápido para a detecção de ruídos tipo sal e pimenta.

\section{MNF utilizando a APC}

A realização de uma etapa prévia de APC para segmentar amostra de ruído é o procedimento mais usual no emprego do MNF. № entanto, para a eliminação dos diferentes tipos de rú́do é importante avaliar suas características de variância e correlação para melhor efetuar sua eliminação. Dessa forma, pode-se estabelecer três principais tipos de ruídos de acordo com suas características de variância e correlação: a) ruídos não-correlacionados, com igual variância em todas as bandas; $b$ ) ruídos altamente correlacionados; e c) ruídos com matriz de covariância desconhecida.

\section{Ruídos não-correlacionados, com igual variância em todas as bandas}

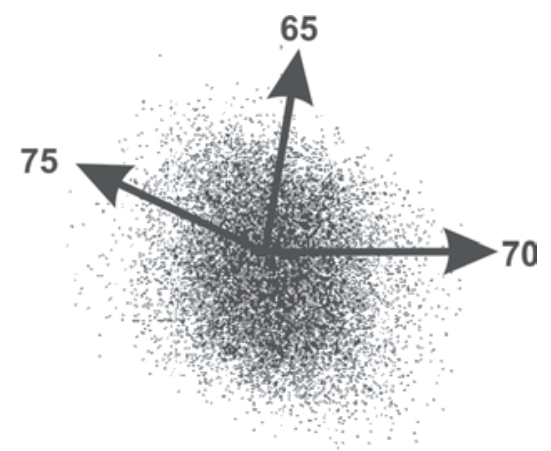

Figura 4 -Distribuição esférica do ruído não-correlacionado, com igual variância em um espaço $\mathrm{n}$-dimensional onde são apresentadas as componentes $65^{a}, 70^{a}$, e $75^{a}$ MNF. Figure 4-Spherical distribution of the non-correlated noise with the same variance in a n-dimensional space where the $65^{h}, 70^{h}$ and $75^{h}$ components are presented.

Esse tipo de ruído caracteriza-se por apresentar uma distribuição esférica em torno de sua média (Figura 4). A APC proporciona um excelente ordenamento desse tipo de ruído. 0 sinal fica retido nas

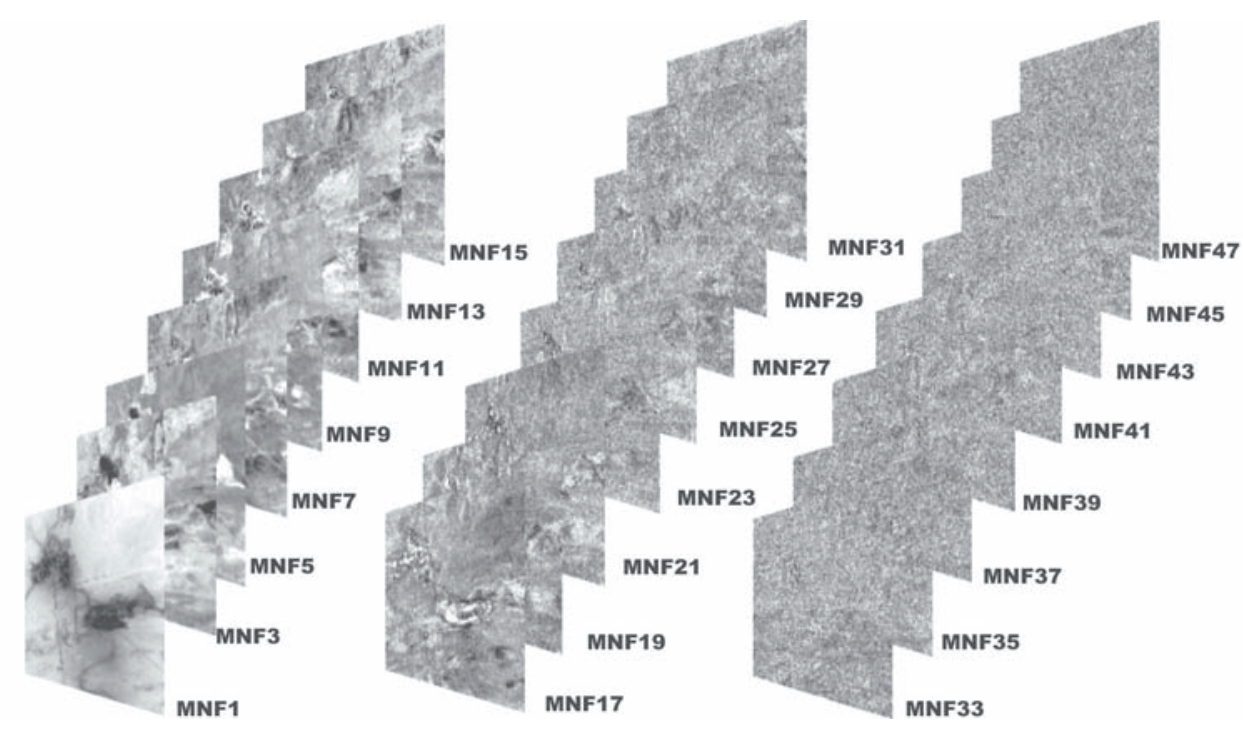

Figura 5 - Degradação do sinal ao longo das componentes MNF.

Figure 5 - Degradation of the signal throughout the MNF components. 
primeiras bandas nos eixos de maior variância, enquanto o ruído tende a ficar nas últimas bandas nos eixos com menor variância e correlação. Dessa forma, o emprego da transformação APC é ideal para segmentar e amostrar esse tipo de ruído para ser empregado no MNF.

Dentro do espaço MNF, a identificação das componentes relativas ao sinal é obtida tanto pelo exame conjunto do gráfico de autovalores como pela qualidade visual das suas componentes. Como exemplificação, a Figura 5 demonstra 0 comportamento das componentes MNF para a região da mina de Niquelândia. Observa-se que o sinal fica agrupado nas primeiras bandas, segregando o ruído para as demais. Essa característica também é evidenciada pelo gráfico de autovalores, que apresenta uma expressiva queda a partir da 15ํㅡㄹ componente (Figura 6).

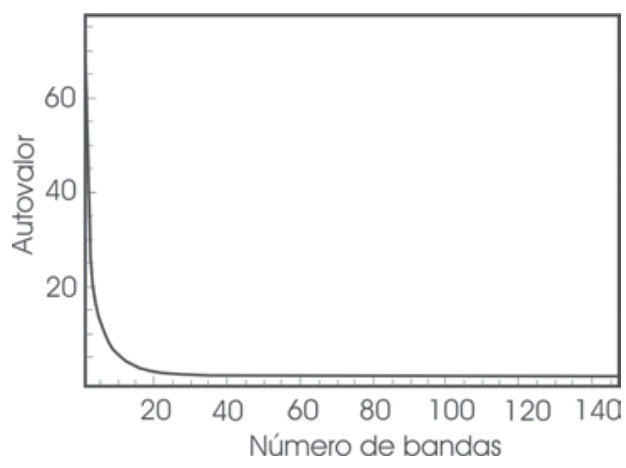

Figura 6 - Gráfico demonstrando o comportamento dos autovalores ao longo das bandas, facilitando a segregação das bandas ruidosas. No presente caso, o limite estabelecido entre o sinal e o ruído foi no ponto de inflexão do gráfico situado no ponto de autovalor igual a 3 que corresponde às 15 primeiras componentes do MNF.

Figure 6-Graphic showing the behavior of eigenvalue throughout the bands, facilitating the segregation of noise bands. In this case, the limit established between the signal and the noise ocurred in the inflexion point of the graphic, located in the eigenvalue point the equals to 3 and corresponds to the 15 first MNF components.

Em geral observa-se uma significativa redução da informação, que passa a adquirir um tamanho de apenas $10 \%$ das bandas iniciais introduzidas. Esse fato deve-se à pronunciada redundância de informação presente nas imagens hiperespectrais, o que é facilmente demonstrado pela alta correlação entre suas bandas (Figura 7).

Os ruídos não-correlacionados de igual variância em todas as bandas são os ruídos mais comuns existentes nas imagens hiperespectrais, 0 que faz do procedimento descrito o método mais empregado. 0 predomínio desse tipo de ruído nas imagens explica o relativo sucesso obtido com a APC para a segmentação do ruído.

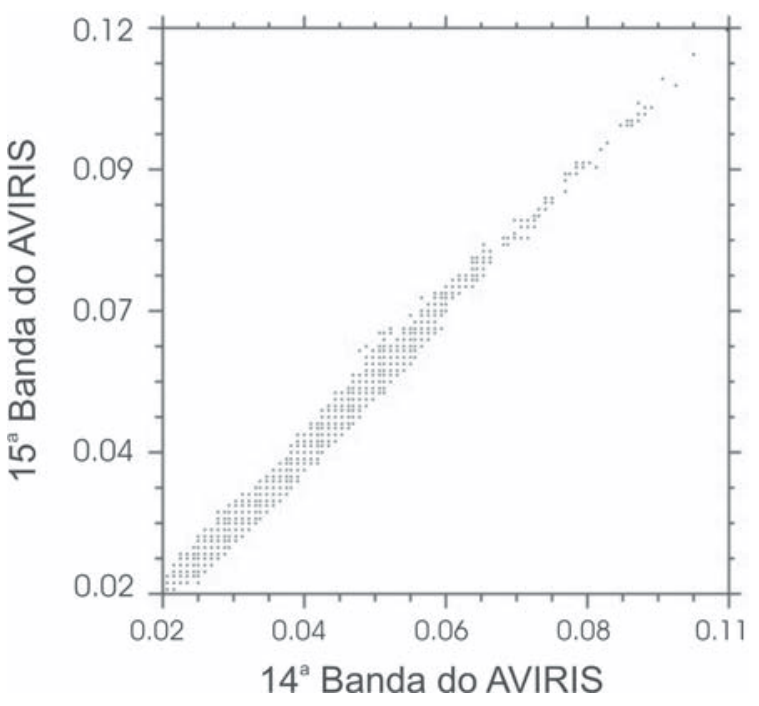

Figura 7 - Gráfico de dispersão entre a $15^{a}$ e $14^{a}$ bandas do AVIRIS. Figure 7 - Graphic of dispersion between the $15^{\text {th }}$ and $14^{\text {hh }}$ AVIRIS bands.

\section{Ruídos altamente correlacionados}

A existência de alta correlação do ruído segundo uma função Yi $(x)$ permite que este se concentre em uma única componente APC ou MNF. Desta forma, a presença de uma combinação linear permite a concentração do ruído em uma banda, tornando as demais bandas livres de ruído. No entanto, a componente do ruído de alta correlação fica junto às componentes de sinais por causa de sua alta variância e correlação. Portanto, deve-se analisar atentamente as componentes referentes ao sinal para detectar sua existência. A luminosidade diferenciada na imagem pode provocar esse tipo de feição.

A transformação MNF na imagem de Niquelândia apresenta esse tipo de ruído na segunda componente, que se caracteriza por uma gradação lateral de tonalidade (Figura 8). Comparado-a com a banda 60, observa-se que tal comportamento não corresponde aos alvos de superfície. Um perfil horizontal na parte norte da $2^{a}$ componente MNF (área sobre vegetação), evidencia uma forte inclinação dos valores (Figura 9) que não correspondem aos alvos imageados.

\section{Ruídos com matriz de covariância desconhecida}

Constituem ruídos de degradação do sinal, como sal e pimenta e linhas danificadas na imagem. Para as imagens do AVIRIS, Hoffman \& Jonhson (1994) identificam um ruído típico proveniente de um ruído 

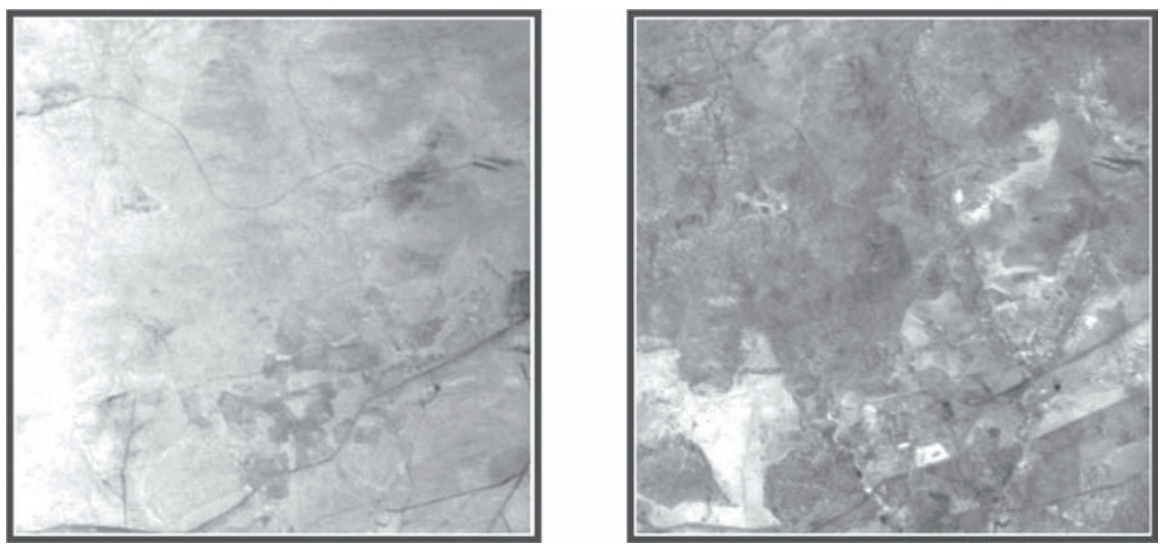

Figura 8 - a) segunda componente MNF para a imagem hiperespectral de Niquelândia que evidencia uma concentração de ruído; e

b) banda 60 do AVIRIS correspondente ao comprimento de onda 0,9242mm.

Figure 8 - a) second MNF component for the hyperspectral image of Niquelandia showing a noise concentration; b) AVIRIS 60 band correspondent to the wave length $0,9242 \mathrm{~mm}$

eletrostático oriundo do instrumento em vôo. Esse ruído é referido como ES e aparece como um pico isolado nos dados. Esse pico é confinado a uma linha, mas pode se estender para dois ou três canais e amostras adjacentes.

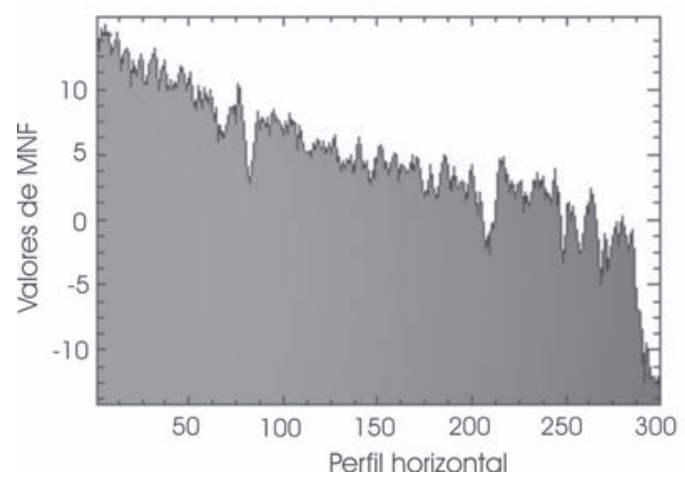

Figura 9 - Perfil horizontal na parte norte da $2^{a}$ componente MNF sobre áreas com cobertura vegetal.

Figure 9- Horizontal profile from the northern part of the 2nd MNF component over areas with vegetal covering.

Os ruídos de degradação do sinal apresentam um comportamento típico no espaço MNF junto às componentes mais ruidosas. A simples visualização, em gráficos de dispersão ou no visualizador n-dimensional das componentes MNF relativas ao ruído evidenciam claramente os pixels com degradação do sinal. Esses exibem um comportamento tíicico, ficando isolados e distanciados da nuvem esférica onde estão agrupados os ruídos de igual variância não-correlacionados. Essa característica deve-se à peculiaridade de sua variância que não condiz nem com o comportamento espectral dos alvos imageados nem com os ruídos não-correlacionados.

Como exemplificaçãa, mostra-se para uma cena da imagem AVIRIS, relativa à região de Niquelândia (GO), o gráfico de dispersão entre a $34^{\circledR}$ e $22^{\circ}$ componentes MNF relativas aos rúdos de igual variância e não-correlacionados (Figura 10). Nesse gráfico é destacada a presença de um pixe/referente ao ruído tipo sal e pimenta posicionado no canto superior esquerdo. A curva espectral desse ponto demonstra a inexistência de uma faixa do espectro que 0 torne diferenciado dos demais pixels.

0 procedimento descrito apresenta vantagens em relaçãa ao método de filtragem proposto por Green e outros (1988). 0 emprego desse procedimento é bem mais rápido e simples, pois permite a detecção dos pixe/s ruidosos de forma simultânea para todas as bandas, ao invés do método de filtragem em que é feita uma varredura banda por banda. Essa característica deve-se ao fato de que, independente da banda que contém o ruído degenerativo, o comportamento descrito na nuvem de variância é sempre diagnóstico.

\section{PROPOSTA DE MÉTODO SEQÜENCIAL PARA ELIMINAÇÃO DOS DIFERENTES TIPOS DE RUÍDOS ATRAVÉS DO MNF}

Diante da necessidade de eliminar diferentes tipos de ruídos em uma mesma imagem, podem-se esquematizar procedimentos com MNF sucessivos. É interessante ressaltar que o MNF deve ser sempre realizado 


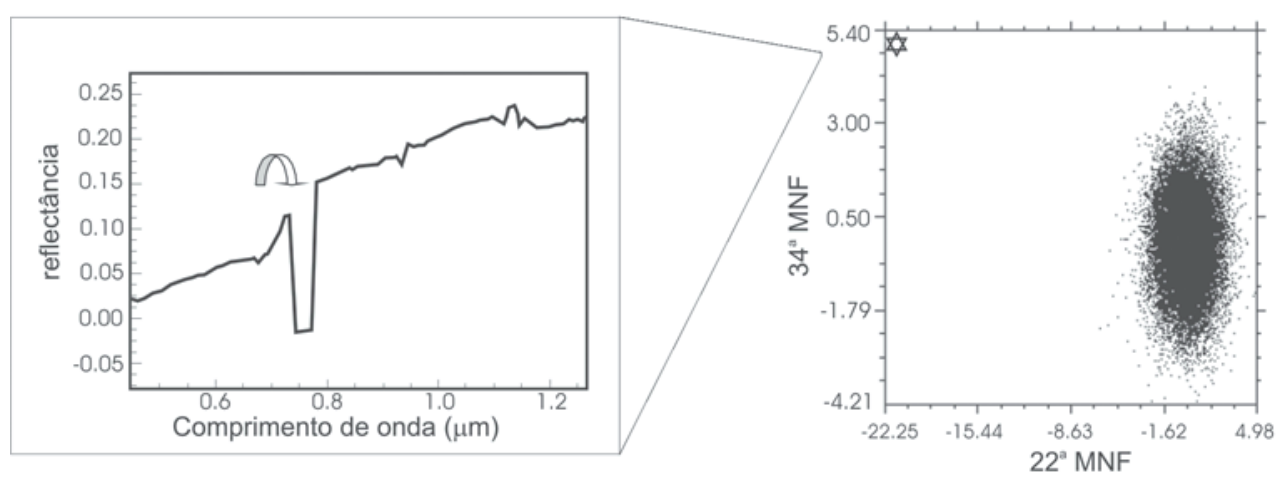

Figura 10 - Determinação de ruído tipo sal e pimenta a partir de um gráfico de dispersão entre componentes MNF relativas à faixa de ruído. Figure 10 - Determination of noise of the salt and pepper type from a graphic of dispersion among MNF components related to the noise band.

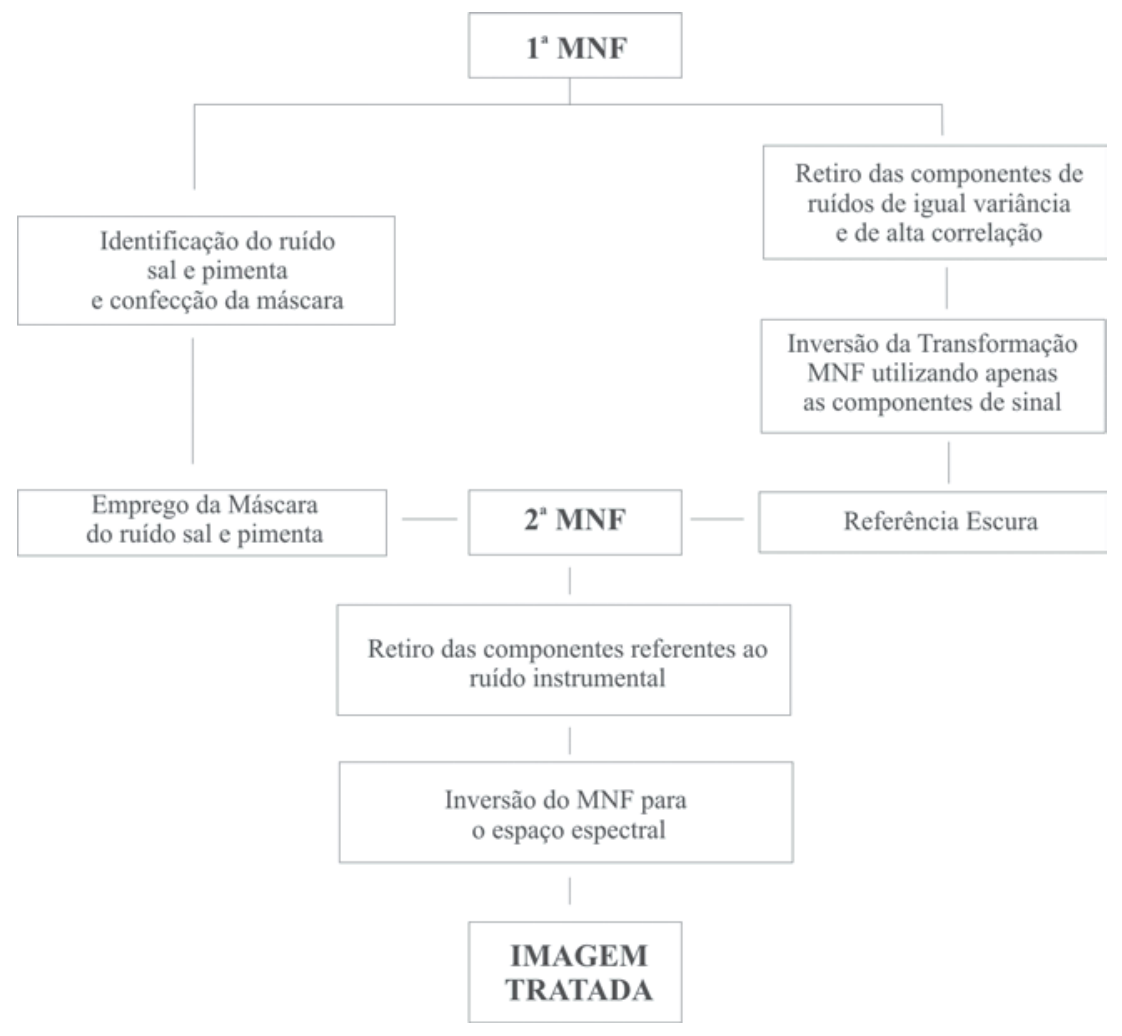

Figura 11 - Procedimento para eliminação do ruído utilizando MNF seqüencial. Figure 11 - Procedures for noise elimination using MNF sequential. 
sobre as bandas de interesse, devendo ser negligenciadas as bandas degradadas pela correçã̃o atmosférica.

Para as imagens AVIRIS foi esquematizado um procedimento seqüencial de MNF, em duas etapas (Figura 11). A primeira etapa do MNF é para segregar os ruídos segundo a sua variância. Após a transformação MNF, os ruídos tipo sal e pimenta devem ser identificados junto às componentes de maior ruído, como foi descrito anteriormente. A partir da seleção, deve-se gerar uma máscara para esses pontos para empregá-la no $2^{2}$ MNF. Efetua-se a inversão do MNF desconsiderando as componentes relativas ao ruído não correlacionado (análise do gráfico de autovalores ao longo das bandas) e o ruído de alta correlação contido nas primeiras bandas.

Sobre a imagem invertida da $1^{a}$ MNF, realiza-se uma segunda etapa de MNF para segregar os ruídos do tipo instrumental (utilizando a referência escura) e do tipo sal e pimenta (utilizando a máscara previamente confeccionada). Procede-se à inversão do $2^{0}$ MNF retirando as componentes do ruído instrumental retidas nas últimas componentes.

A Figura 12 apresenta, para um mesmo ponto, curvas espectrais antes e depois do tratamento seqüencial do MNF. Observa-se em detalhe que 0 espectro sofre uma acentuada suavização com 0 tratamento de remoção do ruído. A remoção dos ruídos é muito importante, pois as feições secundárias oriundas desses ruídos podem atrapalhar os procedimentos de classificação.

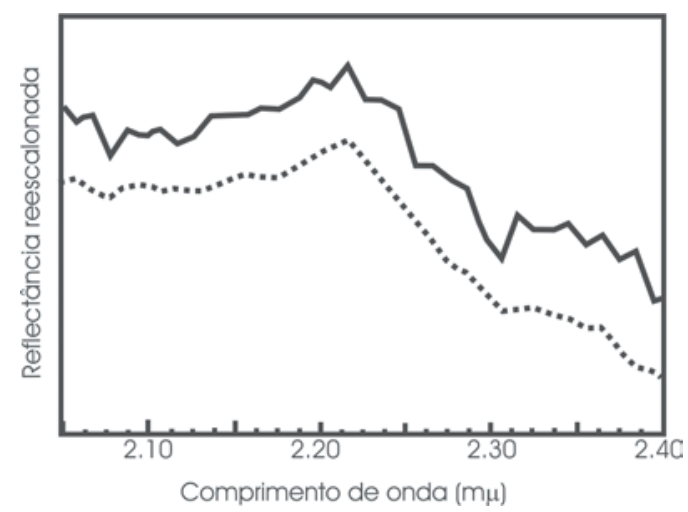

Figura 12 - Comparação de um espectro do sensor AVIRIS: a) antes (linha inteira); e b) após o tratamento com o MNF seqüencial (linha pontilhada).

Figure 12 - Comparison of a spectrum from AVIRIS sensor:

a) before (full line) and b) after treatment with MNF sequential (dotted line).

\section{CONCLUSÃO}

0 tratamento do ruído é uma etapa fundamental para 0 processamentos digital de imagens hiperespectrais, pois delas dependem os demais procedimento para a identificação e quantificação dos alvos superficiais. A complexidade dessa etapa aumenta devido à existência de vários tipos de ruídos que devem ser tratados de formas distintas. Dessa maneira, muitos métodos são desenvolvidos especificamente para um tipo de ruído.

Uma vantagem do método proposto, tratamento seqüencial MNF, advêm da possibilidade de se escolher os principais tipos de ruídos, 0 que proporciona a obtenção de espectros mais fidedignos. Constituído por duas etapas, este método é rápido e de fácil aplicação.

\section{Agradecimentos}

Os autores agradecem a disponibilização das imagens AVIRIS pelo INPE (Instituto Nacional de Pesquisas Espaciais) e NASA (National Aeronautics and Space Administration).

\section{REFERÊNCIAS}

ABRAMS, M. G. Imaging spectrometry: aircraft and space program. In: Int. Geosci. Remote Sensing Symp., 1986, Zurich. Proceedings. Zurich: ESA, 1986. p. 231-234.

BOARDMAN, J. W.; KRUSE, F. A. Automated spectral analysis: a geologic example using AVIRIS data, north Grapevine Mountais, Nevada. In: Thematic Conference on Geologic Remote Sensing, 10., 1994, Ann Arbor. Proceedings. Ann Arbor Ann Arbor: Environmental Research Institute of Michigan, 1994. p. 407-418.

GREEN, A. A.; BERMAN, M.; SWITZER, P.; CRAIG, M. D. A transformation for ordering multispectral data in terms of images quality with implications for noise removal. IEEE Trans. Geosci. Remote Sensing, v. 26, n. 1, p. 65-74, 1988.

GREEN, R. O. et al. Imaging Spectroscopy and the Airborne Visible: infrared imaging spectrometer (AVIRIS). Remote Sens. Environ., v. 65, p. 227248, 1998.

HOFFMAN, R. N.; JONHSON, D. W. Application of EOF's to multispectral imagery: data compression and noise detection for AVIRIS. IEEE Trans. Geosci. Remote Sensing, v. 32, n. 1, p. 25-34, 1994.

HORWITZ, H. M. Estimating the proportions of objects within a single resolution element of a multispectral scanner. In: Int'l Symp. Remote Sens. Environ, 7.,1971, Ann Abor. Proceedings. Ann Abor, 1971. p. 1307-1320. 
KAUFMAN, Y. J. et al. Smoke, clouds, and Radiation-Brazil (SCAR-B) experiment. J. Geophys. Res., v. 103, n. D24. p. 31.783-31.808, 1998. KRUSE, F. A. Use of airborne imaging spectrometer data to map minerals associated with hidrothermally altered rocks in the Northern Grapevine Mountains, Nevada and California, Remote Sens. Environ, v. 24, p. 31 52, 1988.

LEE, J. B.; WOODYATT, A. S.; BERMAN M. Enhancement of high spectral resolution remote sensing data by a noise: adjusted principal components transform. IEEE Trans. Geosci. Remote Sensing, v. 28, n. 3, p. 295$304,1990$.
RICHARDSON, A. J. Plant, soil and shadow reflectance components of row crops. Photogr. Eng. Remote Sens., v. 41, p. 1401-1407, 1975.

ROSE, J. F.. Spatial interference in the AVIRIS imaging spectrometer. Photog. Eng. Remote Sensing, v. 55, n. 9, p. 1.339-1.346, 1989.

VANE G.; GOETZ, A., F. H. Terrestrial imaging spectrometry, Remote Sens. Environ., v. 24, p. 1-29, 1988.

VANE, G. Airborne visible/infrared imaging spectrometer (AVIRIS): an advanced tool for earth remote sensing. IEEE Int. Geosci. Remote Sens. Symp., SP215, New York: IEEE, 1984. p. 751-757.

\section{NOTAS SOBRE OS AUTORES}

Osmar Abílio de Carvalho Júnior recebeu o título de geólogo pela Universidade de Brasília em 1990, e de mestre e doutor em prospecção mineral pela Universidade de Brasília em 1995 e 2000, respectivamente. Atualmente é professor adjunto da Universidade de Braślia, realizando pesquisas sobre o processamento digital de imagens multiespectrais e hiperespectrais.

Ana Paula Ferreira de Carvalho recebeu o título de engenheira agronômica pela Universidade de Braślia em 1992, o grau de mestre em Ecologia pela Universidade de Braślia em 1998 e atualmente realiza o doutorado em imagens hiperespectrais no Departamento de Ecologia da Universidade de Braśília. Trabalha como pesquisadora do laboratório de sensoriamento remoto do Instituto Nacional de Colonização e Reforma Agrária (INCRA).

Paulo Roberto Meneses recebeu o título de geólogo pela Universidade de Brasília em 1973, o grau de mestre pelo Instituto Nacional de Pesquisas Espaciais (INPE) em 1977, e o grau de doutor pela Universidade de São Paulo (USP) em 1986. Atualmente é professor Adjunto do Departamento de Geologia Geral e Aplicada e chefe do Laboratório de Sensoriamento Remoto e Geoprocessamento do Departamento de Geologia Geral e Aplicada. Possui como principais linhas de pesquisa o sensoriamento remoto e geoprocessamento, análise geo-ambiental e integração de dados geofísicos e de imagens.

Renato Fontes Guimarães recebeu o título de engenheiro cartógrafo pela Universidade Estadual do Rio de Janeiro em 1987, 0 grau de mestre em geofísica pelo Observatório Nacional em 1991 e 0 grau de doutor em geologia pela Universidade Federal do Rio de Janeiro em 2000. Atualmente é professor Adjunto do Departamento de Geografia e chefe do Laboratório de Sistemas de Informações Espaciais do Departamento de Geografia. Possui como principais linhas de pesquisa o sensoriamento remoto e a modelagem matemática dos processos erosivos. 